\title{
UAV intelligent system for patrol missions
}

\author{
Pengfei Han ${ }^{1, a}$, Hongwei Zhao ${ }^{2,3, b^{*}}$ and Changzheng Chen ${ }^{3, c}$ \\ ${ }^{1}$ College of electronic science \& engineering, Jilin University, Jilin, Changchun,130012 \\ ${ }^{2}$ Department of computer science and technology, Jilin University, Jilin, Changchun,130012; \\ ${ }^{3}$ State Key Laboratory of applied optics, Changchun, 130033 \\ a1974596222@.com, bhaohw@jlu.edu.cn, may328@163.com
}

Keywords: UAV, patrol, battery life, communication

Abstract. In response to the current UAVs need to man-control, poor life, signal transmission distance limitations can not be caused by the application of the UAV for patrolling, this article for the patrol UAV in the life, route planning, communication proposed a certain program, so that UAVs have more powerful features to meet the drone patrol. In this patrol system, the UAV carries a binocular camera and GPS, and through the image recognition and GPS acquisition of information to identify obstacles to avoid the effect. And then for the UAV designed to automatically replace the battery base station, the base station through the mechanical device for the UAV to replace the battery, and automatically replace the battery down for backup. As for communications, UAVs can use the 4G module, eliminating the need for funds to transmit images, and building a network of multiple base stations to expand the flight range with a base station that only has the ability to change batteries. The experiment proves that the solution can solve the shortcomings of the UAV in life and communication, and the avoidance obstacle function can greatly simplify the operation in the process of using the UAV. As long as people through the host computer to set the patrol route or patrol range, the rest all have procedures to complete automatic control, can be used as patrol unmanned aerial vehicles.

\section{Introduction}

Since December 17, 1903, the world will be brought to the sky an aircraft, with the rapid development of science and technology, multi-rotor UAV came into being, with its small size, simple operation, high reliability, strong function, low cost get rapid development, and more and more people's attention. Major military agencies, research institutes and unmanned aerial vehicles companies at home and abroad have conducted in-depth research on the UAV in military and civil fields ${ }^{[1]}$. Quadrotor UAV by adjusting the four motor speed to achieve the body's attitude adjustment and position changes. Quadrotor is an under-driven, six-degree-of-freedom vertical lift with four inputs but six outputs for vertical, pitching, tumbling, yaw, fore and aft, lateral movements, To meet the basic flight requirements. But the most powerful feature of drones is their ability to carry a variety of external equipment to accomplish various tasks that can help workers accomplish their tasks or reduce their workload. Security has always been the focus of many companies, departments and schools. It is even more difficult for security patrols in areas with large security areas and complex road conditions. The UAV patrol has the advantages of reducing the workload of security personnel, greatly improving the efficiency and not being affected by the terrain and road conditions, but there are also outstanding shortcomings in UAV patrolling, poor power consumption, poor endurance, and signal transmission distance restrictions, high costs and other issues ${ }^{[2]}$ also limits the promotion of patrol UAVs. However, these technical problems do not obscure the advantages of UAS. With the progress of the times, UAV technologies will also continue to mature, and UAVs will surely bloom its proper light ${ }^{[3]}$. 


\section{Patrol System Architecture.}

Shown in Figure 1, the entire system structure can be divided into aerial drone, ground station, security system PC terminal three parts. After man-made patrolling or patrolling routes, the drone began to perform its mission. During the patrolling process, the UAV automatically recognize the obstacle to avoid it and transmit the captured video and UAV location information to the ground station via the wireless transceiver module ${ }^{[4]}$. The ground station then feeds back the information to the security system's PC terminal for corresponding processing, PC-side can also be related to the control of $\mathrm{UAVS}^{[5]}$.

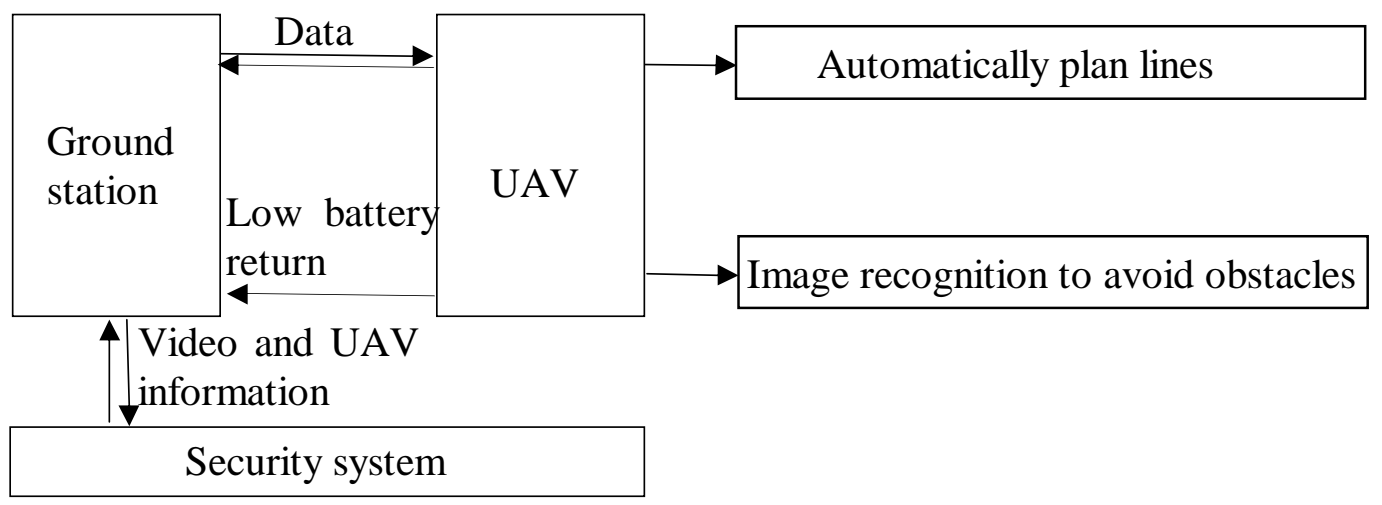

Figure 1 Patrol system architecture

Patrol route planning. UAV to patrol function, patrol route planning is one of the essential and the key one. In the past examples of controlling a UAV by a host computer, due to the complicated environment, most UASs still can not be completely separated from human control. Therefore, a complete system is needed to achieve the goal of liberating manpower. In the actual patrol process, there are many obstacles on the route is the main problem, but also consider the wireless transmission delay issue, so the UAV must be in the process of patrol by airborne MCU real-time identification and avoid obstacles, Obstacles are generally divided into two types of obstacles such as buildings or trees, UAVs can be avoided by image recognition ${ }^{[6]}$ and airborne GPS, the program shown in Figure 2.

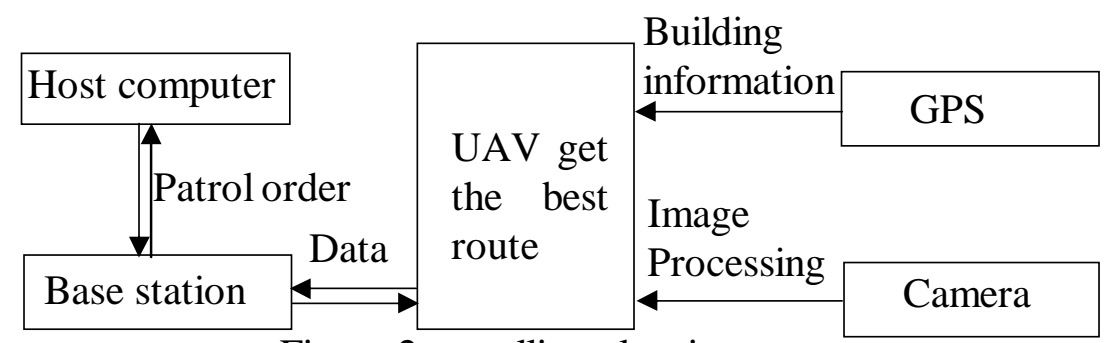

Figure 2 patrolling planning

A program to avoid buildings is to get through the GPS to obtain the map to avoid the other is to take GPS-based image recognition as a secondary method of building over the bypass to avoid and patrolling to the roof of the building that is easy to ignore position. Comparison of the two programs have their own advantages and disadvantages, according to the corresponding topography and the specific circumstances, but for other obstacles can be avoided by image recognition.

Solve the interference of obstacles, you can make the host computer via Bluetooth, WIFI or Ethernet connection to the base station to issue commands to the drone. Patrol program can be used to set patrol routes for the UAV or set the patrol range ${ }^{[7]}$, which set the patrol range for the UAV should be controlled by the program to make it as much as possible to improve patrol efficiency, patrol routes and biased in favor of coverage monitoring and monitoring $o$ and easy accident area.

Life. $\mathrm{n}$ the practical application of the UAV, poor endurance has always been the biggest problem with the UAV, and most UAVs can only fly for about 20 minutes even at full capacity. UAVs are generally increased in flight time by increasing the energy density of the drone, but no matter how high 
the energy density is, it is still impossible for the UAV to fly for an indefinite period of time, patrolling the deadline even if the battery is returned to the point of departure for battery replacement, to this end can be solved from two aspects of the life of the UAV:

(1) It is possible to design a base station that can replace a battery by a mechanical device for the $\mathrm{UAV}^{[8]}$. As shown in Figure 3, the UAV continuously detects the battery level and calculates the time until the remaining capacity can fly and the distance to the nearest base station of the time, return to the base station when the battery is low to replace the battery ${ }^{[9]}$, the base station will replace the battery under charge, the UAV continues to take off to continue the task.

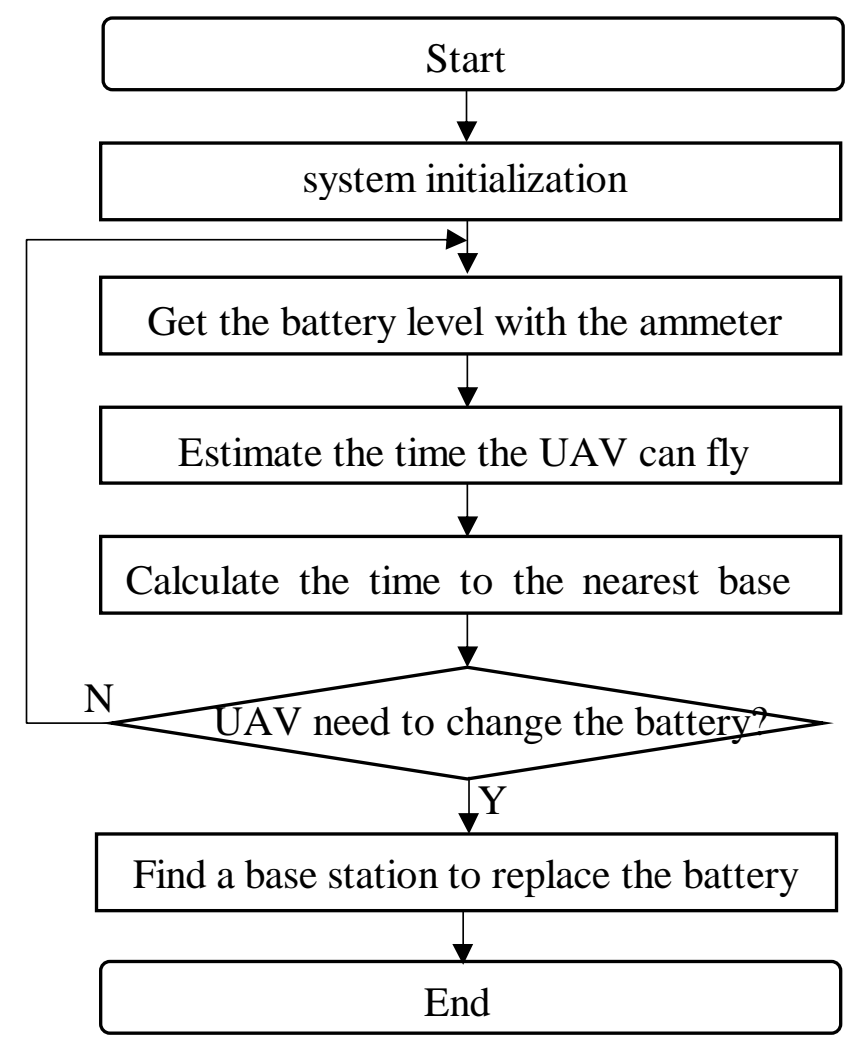

Figure 3 automatically replace the battery flow

(2)Since the battery charge time is much longer than the UAV flight time, a larger number of batteries may be required as a basis simply by replacing the battery and charging the replaced battery, which would increase the cost of the UAV. As the UAV to increase the battery capacity will increase the weight of the UAV will increase power consumption, the actual length of the flight did not increase a lot, in vain to increase the cost of many, so need to reduce the weight of the drone work hard and use low speed brushless DC motor and large propeller, in order to reduce power consumption to improve the purpose of flying time, so as to minimize the number of rotating battery, but also can reduce the frequency of battery replacement, improve efficiency. However, the research of new battery technology is fiery. With the development of science and technology, the battery will eventually make breakthrough progress. By then, the problem of the life of the UAV will be solved.

Communication. In the mobile phone technology is quite mature today, mobile $4 \mathrm{G}$ has entered the lives of people, and even 5G have already begun to deploy. $4 \mathrm{G}$ is a set of $3 \mathrm{G}$ and WLAN in one, can quickly transfer data, high quality, audio, video and images. 4G to $100 \mathrm{Mbps}$ above the speed of download $^{[10]}$, to meet the UAV transmission of video, signal for wireless service requirements. In recent years, the state has also promulgated the relevant policies of "reducing the speed of fee increases." Recently, operators also introduced unlimited traffic packages so that the unmanned aerial vehicles can be equipped with $4 \mathrm{G}$ modules to transmit wirelessly through the $4 \mathrm{G}$ network of operators.

As shown in Figure 4, in the traditional mode, the aerial camera transmits the video signal to the ground end through the image transmission. However, the image transmission mode uses low delay and low price of the analog signal, but the image quality is low. The use of digital signals, although the 
image quality is high, but the signal delay high, the price is high. And the use of image transmission equipment, most of the transmission distance within $2 \mathrm{~km}$. If using $4 \mathrm{G}$ network for signal transmission, As shown in Figure 5, using 4G network transmission not only low latency, fast, and no transmission distance requirements, can greatly expand the scope of work. Digital video transmission equipment capable of transmitting high-definition video stream transmission most of the expensive equipment, the basic price is even higher in the thousand, and a 4G module is only a few hundred dollars, even with the use of the operator monthly rent, relative to the more High-price digital module also has price advantage.

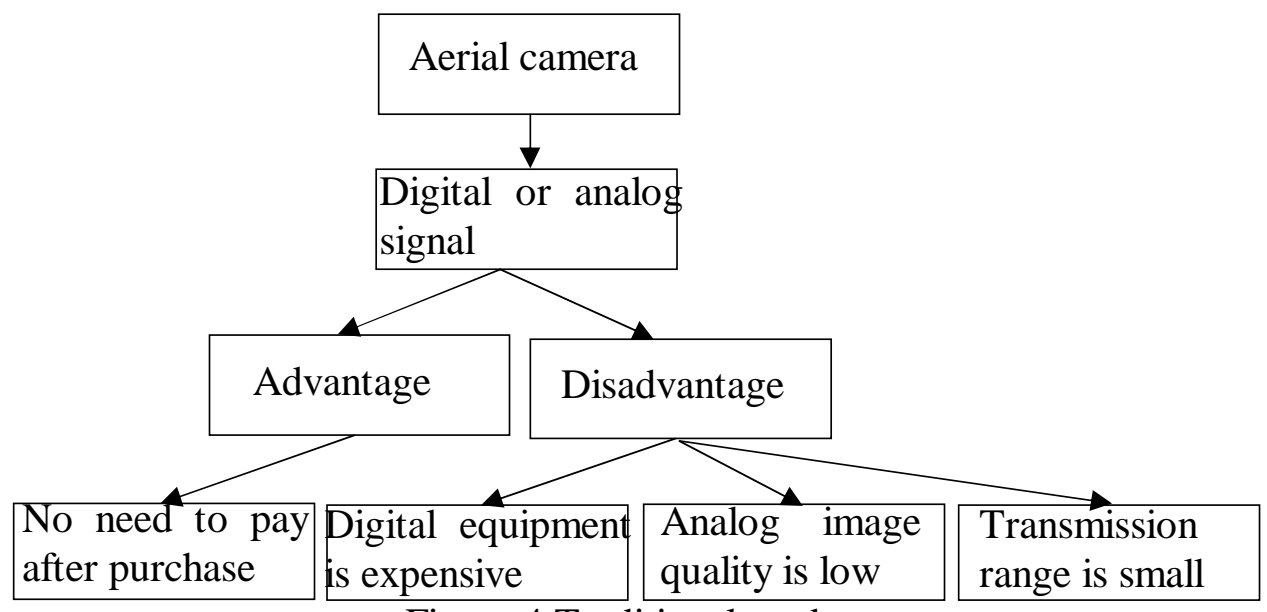

Figure 4 Traditional mode

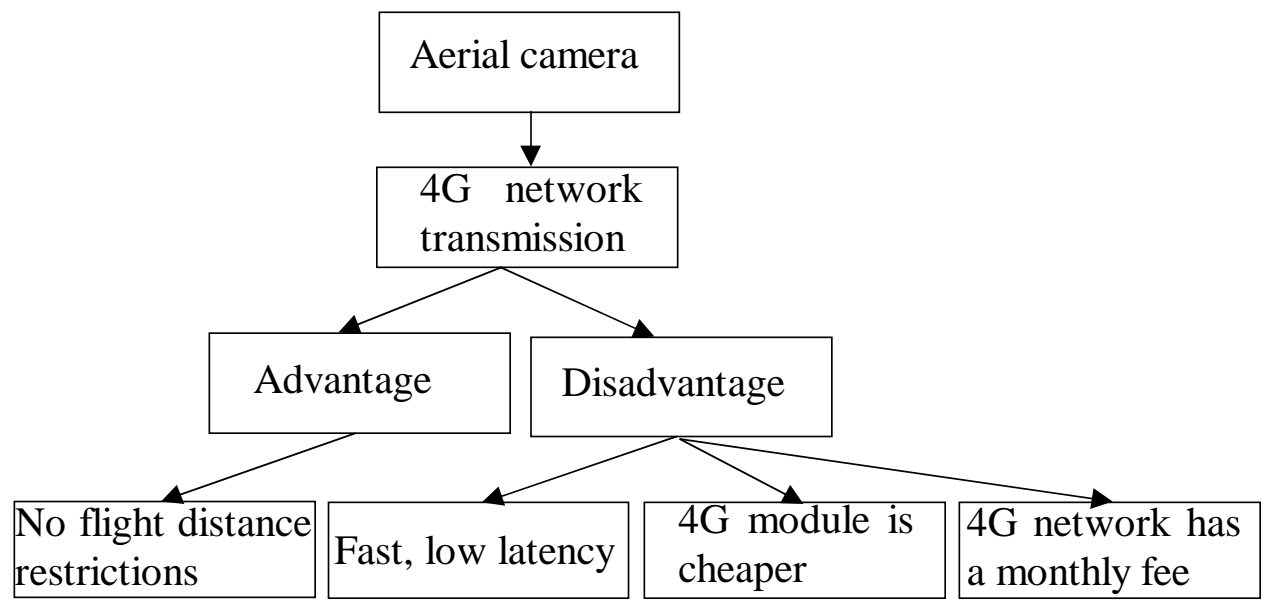

Figure 5 uses $4 \mathrm{G}$ network

\section{Experiments}

Experiments show that this solution is feasible and can solve the problem that the UAV needs to be frequently charged or replaced due to the short flight time and has no flight distance limitation when the power is enough to return to the base station. Operators can be completely controlled on the host computer, even if novices only need to know some basic knowledge can be completely manipulated, novice friendly. To meet the design requirements, to some extent to solve the UAV in life, signal, operation and other aspects of the existing problems.

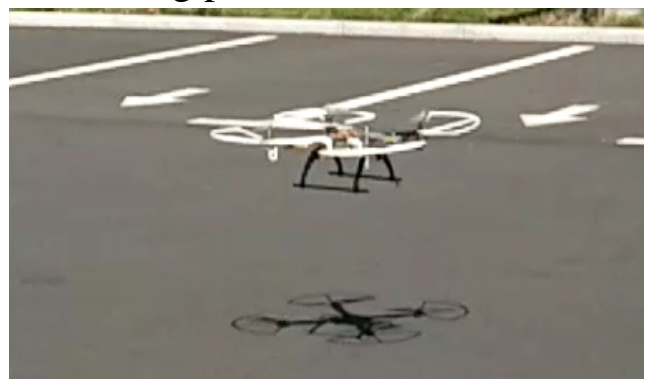

Figure 6 UAV flight chart 


\section{Conclusion}

Patrol drone has many applications, such as large-scale forest fire prevention project, rescue and rescue work, all aspects of the need for protection. Patrol UAVs to reduce the problems of life based on not only in the patrol can also be applied to the tasks that require continuous flight work, such as television recording, you can reduce the working hours, shooting in a shorter period of time more videos; patrol drones can be dispatched for exploration in the most dangerous situations or in areas that people can not directly view.

Such a solution can reduce the manpower burden, and in some more urgent situations, enable relevant personnel to devote more energy to other matters rather than waste a lot of time on these relatively basic operations. This can greatly improve the efficiency of the work, the UAV life can not be completely solved the case, but also a temporary solution.

\section{Acknowledgements}

The corresponding author is Zhao Hongwei. The authors are grateful to the anonymous reviewers for their insightful comments which have certainly improved this paper.

This work was supported in part by the National Natural Science Foundation of China under Grant 61101155, Jilin province development and Reform Commission Special industrial innovation 2016 C035 and State Key Laboratory of applied optics.

\section{References}

[1] J.M. Sullivan: Revolution or evolution? The rise of the UAVs. Technology and Society. Weapons and Wires: Prevention and Safety in a Time of Fear. ISTAS 2005. Proceedings. 2005 International Symposium on(2005).

[2] Andrew, Renault. A Model for Assessing UAV System Architectures [J]. Procedia Computer Science, 2015, (61): 160-167.

[3] Nathan Richard.Advantages and Challenges of Unmanned Aerial Vehicle Autonomy in the Postheroic Age[D]. Virginia: James Madison University.2012.

[4] Zhang, Wen, Qin, Guo-Shun. Design of UAV Ground Control Station[C]. Poland:Prof. Janusz Kacprzyk, 2012. 249-256.

[5] Han Wang,Bingjing Yan,Xiaoxia Li,Xuejing Luo,Qiang Yang,Wenjun Yan: On optimal path planning for UAV based patrolling in complex 3D topographies.Information and Automation (ICIA), IEEE International Conference on, pp. 986-99,2016.

[6] Brandon, R, Call. OBSTACLE AVOIDANCE FOR UNMANNED AIR VEHICLES[C]. Provo:Brigham Young University, 2006.

[7] P, DohertyP, HaslumF, HeintzT, MerzP, NyblomT, PerssonB, Wingman. A Distributed Architecture for Autonomous Unmanned Aerial Vehicle Experimentation[C]. Sweden:Dept.of Computer and Information Science, 2007. 233-242.

[8] Swieringa, K.A., et al.: Autonomous battery swapping system for small-scale helicopters. In: IEEE International Conference on Robotics and Automation (ICRA), pp. 3335-3340 (2010).

[9] Kemper F P, Suzuki K A, Morrison J R. UAV Consumable Replenishment: Design Concepts for Automated Service Stations[J]. Journal of Intelligent \& Robotic Systems, 2011, 61(1-4):369-397.

[10] Shah Faisal. Performance Analysis of 4G Networks [D]. Sweden: Shah Faisal Blekinge Institute.2010. 